\title{
The high-order cyclotron modes in Fermi liquid of Q2D layered conductors
}

\author{
O.V.Kirichenko, V.G.Peschansky, D.I.Stepanenko \\ B.I.Verkin Institute for Low Temperature Physics and Engineering, \\ National Academy of Sciences of the Ukraine, \\ 47 Lenin ave, Kharkiv, 61103, Ukraine
}

Received July 18, 2005

\begin{abstract}
The propagation of electromagnetic waves in layered conductors at the presence of an external magnetic field is investigated theoretically. At certain orientations of a magnetic field concerning the layers of the conductor the collisionless absorption is absent and weakly damping collective modes can propagate even under the strong spatial dispersion. In a short-wave limit the existence of electromagnetic waves with frequencies near the cyclotron resonances is possible at an arbitrary orientation of the wave vector with respect to an external magnetic field. We have obtained the spectrum of waves with the frequencies near the cyclotron resonances of high order with regard to the Fermi-liquid interaction of the electrons.
\end{abstract}

Key words: layered conductors, Fermi liquid, cyclotron waves

PACS: $72.15 . \mathrm{Nj}, 72.30 .+q$

Experimental studies of galvanomagnetic phenomena in a large family of tetrathiafulvalene based ion-radical salts of the form (BEDT - TTF $)_{2} \mathrm{X}(\mathrm{X}$ stands for a set of various anions) indicate that these layered compounds possess the metal type electrical conductivity. This permits to describe electron processes in such conductors based on the well-developed concept of quasiparticles, analogous to conduction electrons in metals. Observation of Shubnikov-de Haas magnetoresistance oscillations prove that the approximation of free path time $\tau$ in these layered conductors can be sufficient for charge carriers to manifest their dynamic properties. Their cyclotron frequency $\Omega$ may significantly exceed $\tau^{-1}$ (see, for example, [1-3] and citations therein).

The sharp anisotropy of the conductivity of layered conductors is apparently connected with the anisotropy of the velocities on the Fermi surface and restricts the choice of suitable models for the Fermi surface. The model of the Fermi surface in the form of a weakly corrugated cylinder is in a good agreement with the experimental investigations of many layered conductors. The results of calculations based on this model are in complete agreement with the experimentally observed 
Shubnikov-de Haas oscillations in the tetrathiafulvalene salts (BEDT - TTF $)_{2} \mathrm{JBr}_{2}$ and $(\mathrm{BEDT}-\mathrm{TTF})_{2} \mathrm{~J}_{3}$.

The charge carrier energy $\varepsilon(\mathbf{p})$ in layered conductors weakly depends on the momentum projection $p_{z}=\mathbf{p n}$ on the normal $\mathbf{n}$ to the layers. The electron energy spectrum can be represented as the Fourier series with respect to the variable $p_{z} / p_{0}$ with rapidly decreasing coefficients. Confining ourselves to the zeroth and first harmonics and neglecting the anisotropy in the layer-plane we can write the dispersion law as follows:

$$
\varepsilon(\mathbf{p})=\frac{p_{x}^{2}+p_{y}^{2}}{2 m}-\varepsilon_{0} \cos \frac{p_{z}}{p_{0}} .
$$

Here $\varepsilon_{0}=\eta v_{\mathrm{F}} p_{0}, v_{\mathrm{F}}^{2} \equiv 2 \varepsilon_{\mathrm{F}} / m, m$ is the effective mass in the layer-plane, $\hbar / p_{0}$ is the distance between the layers, $\hbar$ is the Planck constant, $\varepsilon_{\mathrm{F}}$ is the Fermi energy. The parameter $\eta$ characterizes the anisotropy of the charge carrier energy spectrum. In the absence of a magnetic field the ratio of the conductivity across the layers to the in-plane conductivity is about $\eta^{2}$.

The experimental studies of the propagation of electromagnetic waves in the layered conductors has also attracted the attention of the researches. Several papers appeared, in which the results of the observation of cyclotron resonance in $\alpha-(\mathrm{BEDT}-\mathrm{TTF})_{2} \mathrm{KHg}(\mathrm{SCN})$ and in $\left.(\mathrm{BEDT}-\mathrm{TTF})_{2} \mathrm{ReO}_{4}\left(\mathrm{H}_{2} \mathrm{O}\right)\right)$ have been reported $[4-6]$.

The kinetic phenomena in alternating fields contain rich information about the electron energy spectrum and relaxation properties of charge carriers. In the presence of a high external magnetic field $\mathbf{H}_{0}$ the other types of weakly attenuating electromagnetic waves (helicoidal, magnetohydrodynamic and cyclotron waves) occur. The spectrum of their high frequency branches depends essentially on the constants of Fermi-liquid interaction. The results of the studies of the wave processes in conventional metals with regard to the Fermi liquid interaction of charge carriers, are reported in the monograph [7]. Herein below we consider the propagation of electromagnetic waves in a Fermi liquid of the layered conductors placed in an external magnetic field. The specific features of the quasi-two dimensional electron energy spectrum of a layered conductor manifest themselves in its kinetic properties. The velocity $v_{H}$ of the charge carriers drift along the magnetic field direction gets zero for some values of the angle $\vartheta$ between $\mathbf{H}_{\mathbf{0}}=\left(H_{0} \sin \vartheta, 0, H_{0} \cos \vartheta\right)$ and the normal $\mathbf{n}$ to the layers. This is the condition when there is no collisionless absorption of the electromagnetic wave energy by electrons and weak damping waves can propagate even under the strong spatial dispersion [8]. In a short-wave limit the existence of electromagnetic waves with frequencies near the cyclotron resonance is possible at an arbitrary orientation of the wave vector $\mathbf{k}$ with respect to $\mathbf{H}_{\mathbf{0}}$. We analyze the spectrum of cyclotron waves under the strong spatial dispersion with regard to the Fermi-liquid interaction.

Kinetic properties of the system of fermions should be described by means of the kinetic equation for the density matrix $\hat{\rho}$. In the quasiclassical case when $\hbar \Omega \lesssim$ $T \ll \eta \varepsilon_{\mathrm{F}}$ the quantization of the charge carriers energy in the magnetic field does not essentially affect the magnetization $\mathbf{M}$ ( $T$ is the temperature). Under these 
conditions the density matrix $\varrho$ can be presented as an operator in the space of spin variables and as a function depending on coordinates and momentum.

The interaction between electrons results in the correction to their energy

$$
\delta \hat{\varepsilon}(\mathbf{p}, \mathbf{r}, t)=\operatorname{Tr}_{\sigma^{\prime}} \int \frac{\mathrm{d}^{3} p^{\prime}}{(2 \pi \hbar)^{3}} \Lambda\left(\mathbf{p}, \hat{\boldsymbol{\sigma}}, \mathbf{p}^{\prime}, \hat{\boldsymbol{\sigma}}^{\prime}\right) \delta \hat{\rho}\left(\mathbf{p}, \mathbf{r}, \hat{\boldsymbol{\sigma}}^{\prime}, t\right)
$$

which can be described with the aid of the correlation function $[9,10]$

$$
\Lambda\left(\mathbf{p}, \hat{\boldsymbol{\sigma}}, \mathbf{p}^{\prime}, \hat{\boldsymbol{\sigma}}^{\prime}\right)=L\left(\mathbf{p}, \mathbf{p}^{\prime}\right)+S\left(\mathbf{p}, \mathbf{p}^{\prime}\right) \hat{\boldsymbol{\sigma}} \hat{\boldsymbol{\sigma}}^{\prime}
$$

where $\delta \hat{\rho}$ is the nonequilibrium correction to the density matrix, $\boldsymbol{\sigma}$ are Pauli matrices. The second term on the right-hand part of (3) corresponds to the exchange interaction between electrons.

The alternating electric $\mathbf{E}$ and magnetic $\mathbf{H}^{\sim}$ fields produced by the electric current

$$
\mathbf{j}(\mathbf{r}, t)=\operatorname{eTr}_{\sigma} \int \frac{\mathrm{d}^{3} p}{(2 \pi \hbar)^{3}} \hat{\rho}(\mathbf{p}, \mathbf{r}, \hat{\boldsymbol{\sigma}}, t) \frac{\partial \hat{\varepsilon}}{\partial \mathbf{p}}+\mathrm{c} \mu_{0} \operatorname{rot} \operatorname{Tr}_{\sigma} \int \frac{\mathrm{d}^{3} p}{(2 \pi \hbar)^{3}} \hat{\boldsymbol{\sigma}} \hat{\rho}(\mathbf{p}, \mathbf{r}, \hat{\boldsymbol{\sigma}}, t)
$$

should be determined from the Maxwell equations

$$
\operatorname{rot} \mathbf{H}^{\sim}=\frac{4 \pi}{\mathrm{c}} \mathbf{j}+\frac{1}{\mathrm{c}} \frac{\partial \mathbf{E}}{\partial t}, \quad \operatorname{rot} \mathbf{E}=-\frac{1}{\mathrm{c}} \frac{\partial \mathbf{H}^{\sim}}{\partial t},
$$

where $\mathrm{e}$ is the electron charge, $\mathrm{c}$ is the velocity of light.

Instead of the density matrix $\hat{\rho}$, it will be more convenient to use the distribution function $f(\mathbf{r}, \mathbf{p}, t)=\operatorname{Tr}_{\sigma} \hat{\rho}$ and the spin density $\mathbf{g}(\mathbf{r}, \mathbf{p}, t)=\operatorname{Tr}_{\sigma}(\hat{\boldsymbol{\sigma}} \hat{\rho})$. The function $\mathbf{g}(\mathbf{r}, \mathbf{p}, t)$ and the second term on the right-hand part of (4) describe paramagnetic spin waves predicted by Silin [11] and observed in isotropic metals by Dunifer and Schultz [12]. The properties of spin waves are determined by the magnetic susceptibility tensor $\chi_{i k}(\omega, k)$. For the frequencies, that do not coincide with the frequency of eigen-oscillations of the spin density, the components $\chi_{i k}(\omega, k)$ are of the order of the static paramagnetic susceptibility $\chi_{0} \simeq \mu_{0}^{2} \nu\left(\varepsilon_{\mathrm{F}}\right) \sim 10^{-6}\left(\nu\left(\varepsilon_{\mathrm{F}}\right)\right.$ is the density of states at the Fermi level). For this reason we neglect the spin magnetism of the media, when considering the electromagnetic modes arising from the oscillations of the electron distribution function.

Let us present the distribution function $f$ in the form $f(\mathbf{r}, \mathbf{p}, t)=f_{0}(\varepsilon)-$ $\mathrm{e} \boldsymbol{\Psi}(\mathbf{r}, \mathbf{p}, t) \mathbf{E} \cdot \partial f_{0} / \partial \varepsilon$, where $f_{0}(\varepsilon)$ is the equilibrium Fermi function. The nonequilibrium correction $\boldsymbol{\Psi}(\mathbf{r}, \mathbf{p}, t)$ satisfies the following kinetic equation

Here

$$
\frac{\partial \Psi}{\partial t}+\left(\mathbf{v} \frac{\partial}{\partial \mathbf{r}}+\frac{e}{c}\left(\mathbf{v} \times \mathbf{H}_{0}\right) \frac{\partial}{\partial \mathbf{p}}\right) \boldsymbol{\Phi}=\mathbf{v}+I_{\text {coll }}(\boldsymbol{\Phi})
$$

$$
\boldsymbol{\Phi} \equiv \boldsymbol{\Psi}+\langle L \boldsymbol{\Psi}\rangle, \quad\langle L \boldsymbol{\Psi}\rangle \equiv \int \frac{2 \mathrm{~d}^{3} p^{\prime}}{(2 \pi \hbar)^{3}}\left(-\frac{\partial f_{0}\left(\varepsilon^{\prime}\right)}{\partial \varepsilon^{\prime}}\right) L\left(\mathbf{p}, \mathbf{p}^{\prime}\right) \boldsymbol{\Psi}\left(\mathbf{r}, \mathbf{p}^{\prime}, t\right),
$$

$I_{\text {coll }}$ is the collision integral. 
The wave process can be regarded as harmonic and the coordinate and time dependencies of the fields $\mathbf{E}, \mathbf{H}^{\sim}$ and of the function $\boldsymbol{\Psi}$ can be represented as $\exp (\mathrm{ikr}-\mathrm{i} \omega t)$. Regardless of the weak current of spin magnetization in (4), the current density in a conductor can be written as follows:

$$
j_{i}=\mathrm{e}^{2} \int \frac{2 \mathrm{~d}^{3} p}{(2 \pi \hbar)^{3}}\left(-\frac{\partial f_{0}}{\partial \varepsilon}\right) v_{i} \Phi_{j} E_{j}=\sigma_{i j}(\omega, \mathbf{k}) E_{j},
$$

where $\sigma_{i j}(\omega, \mathbf{k})$ is the electrical conductivity tensor, $\mathbf{v}=\partial \varepsilon(\mathbf{p}) / \partial \mathbf{p}$. Substituting $\mathbf{E}, \mathbf{H}^{\sim}$ in the form of a harmonic wave into the Maxwell equations (5) we obtain the dispersion equation

$$
\operatorname{det}\left[k^{2} \delta_{i j}-k_{i} k_{j}-\frac{\omega^{2}}{c^{2}} \varepsilon_{i j}(\omega, \mathbf{k})\right]=0
$$

which determines the spectrum $\omega(\mathbf{k})$ for the electromagnetic field oscillations. Here $\varepsilon_{i j}(\omega, \mathbf{k})=\delta_{i j}+4 \pi \mathrm{i} \omega^{-1} \sigma_{i j}(\omega, \mathbf{k})$ is the dielectric tensor. We shall use the reference frame where the wave vector $\mathbf{k}=(k \sin \phi, 0, k \cos \phi)$ is oriented in the $x z$ plane. For frequencies much less than the plasma frequency $\omega_{\mathrm{p}}$ the first term in the expression for $\varepsilon_{i j}$ can be neglected.

In the lowest order approximation about the small parameter $\eta$ the function $L\left(\mathbf{p}, \mathbf{p}^{\prime}\right)$ does not depend on $p_{H}=\left(\mathbf{p} \mathbf{H}_{0}\right) / H_{0}$ and can be presented as

$$
L\left(\mathbf{p}, \mathbf{p}^{\prime}\right)=\sum_{n=-\infty}^{\infty} L_{n}(\varepsilon) \mathrm{e}^{i n\left(\varphi-\varphi^{\prime}\right)}
$$

We have chosen the integrals of motion of an electron in a magnetic field $\varepsilon, p_{H}$ and the phase $\varphi=\Omega t_{1}$ at its orbit in the magnetic field as variables in the $\mathbf{p}$-space. Here $t_{1}=\left(\mathrm{c} / \mathrm{e} H_{0}\right) \int \mathrm{d} l / v_{\perp}$ is the time of motion along the trajectory $\varepsilon(\mathbf{p})=\varepsilon_{\mathrm{F}}, p_{H}=$ const, $\mathrm{d} l=\left(\mathrm{d} p_{x}^{2}+\mathrm{d} p_{y}^{2}\right)^{1 / 2}, v_{\perp}$ is the velocity component orthogonal to the external magnetic field [13]. Due to the symmetry of the function $\Lambda\left(\mathbf{p}, \hat{\boldsymbol{\sigma}}, \mathbf{p}^{\prime}, \hat{\boldsymbol{\sigma}}^{\prime}\right)$ with respect to its arguments, the coefficients in (9) satisfy the condition $L_{n}=L_{-n}$. Allowing for next-order terms of the expansion for the correlation function about $\eta$ does not lead to the noticeable correction of the results.

Expanding the functions $\boldsymbol{\Phi}$ and $\boldsymbol{\Psi}$ into a Fourier series with respect to $\varphi$ and equalizing coefficients at $\exp (i n \varphi)$ in the equality $\boldsymbol{\Phi}=\boldsymbol{\Psi}+\langle L \boldsymbol{\Psi}\rangle$, we obtain

$$
\Psi=\boldsymbol{\Phi}-\sum_{n=-\infty}^{\infty} \lambda_{n} \overline{\boldsymbol{\Phi}}^{(n)} \mathrm{e}^{i n \varphi}
$$

Here

$$
\overline{\boldsymbol{\Phi}}^{(n)}=\frac{1}{(2 \pi)^{2}} \int_{0}^{2 \pi} \mathrm{d} \varphi \mathrm{e}^{-i n \varphi} \int_{-\pi}^{\pi} \mathrm{d} \beta \boldsymbol{\Phi}\left(\varepsilon_{\mathrm{F}}, \beta\right) \equiv\left\langle\mathrm{e}^{-i n \varphi} \boldsymbol{\Phi}\right\rangle_{\beta, \varphi}
$$

is the Fourier component for the function $\langle\boldsymbol{\Phi}\rangle_{\beta}, \beta=p_{H} / p_{0} \cos \vartheta,\langle\cdots\rangle_{\beta}=1 / 2 \pi \times$ $\int_{-\pi}^{\pi} \mathrm{d} \beta \cdots, \lambda_{n}=L_{n}^{\sim} /\left(1+L_{n}^{\sim}\right), L_{n}^{\sim}=\nu\left(\varepsilon_{\mathrm{F}}\right) L_{n}$. Substituting (10) into the equation 
(6) and using the equality $\partial / \partial t_{1}=(e / c)\left(\mathbf{v} \times \mathbf{H}_{0}\right) \partial / \partial \mathbf{p}$ we find the kinetic equation for the perturbation of the renormalized distribution function for electrons with the quasi-two-dimensional dispersion law [14]:

$$
\frac{\partial \boldsymbol{\Phi}}{\partial \varphi}-\frac{\mathrm{i}}{\Omega}(\omega-\mathbf{k v}) \boldsymbol{\Phi}=\frac{\mathbf{v}}{\Omega}-\frac{\mathrm{i} \omega}{\Omega} \sum_{n=-\infty}^{\infty} \lambda_{n} \overline{\boldsymbol{\Phi}}_{n}(\varepsilon) \mathrm{e}^{i n \varphi}+\frac{1}{\Omega} I_{\text {coll }}(\boldsymbol{\Phi})
$$

The collision integral in the $\tau$-approximation can be written as $I_{\text {coll }}(\boldsymbol{\Phi})=-\tau^{-1} \boldsymbol{\Phi}$ ( $\tau$ is the relaxation time for the momentum ). Below we consider the wave processes in the range of frequencies satisfying the condition

$$
\omega_{\mathrm{p}} \gg \omega \gg \tau^{-1},
$$

where the asymptotic expression for the spectrum of collective modes does not essentially depend on the concrete form of the collision integral. Here $\omega_{\mathrm{p}}=\left(4 \pi \mathrm{e}^{2} n_{0} / m\right)^{1 / 2}$ is plasma frequency, $n_{0}$ is charge carrier density.

In the first order in $\eta$, the components of electron velocity are easily found by means of standard method of nonlinear mechanics

$$
\begin{aligned}
& v_{x}\left(t_{1}\right)=v_{x}^{(0)}\left(t_{1}\right)+v_{x}^{(1)}\left(t_{1}\right), \quad v_{x}^{(0)}\left(t_{1}\right)=v_{\perp} \cos \Omega(\beta) t_{1}, \quad v_{y}^{(0)}\left(t_{1}\right)=-v_{\perp} \sin \Omega(\beta) t_{1}, \\
& v_{x}^{(1)}\left(t_{1}\right)=\eta v_{\mathrm{F}} \tan \vartheta \mathrm{J}_{0}(\alpha) \sin \beta-\eta v_{\mathrm{F}} \tan \vartheta \sum_{n=2}^{\infty} \frac{\mathrm{J}_{n}(\alpha) \sin (\beta-n \pi / 2)}{n^{2}-1} \cos n \Omega(\beta) t_{1}, \\
& v_{z}\left(t_{1}\right)=\eta v_{\mathrm{F}} \sin \left(\beta-\alpha \cos \Omega(\beta) t_{1}\right) .
\end{aligned}
$$

Here

$$
\Omega(\beta)=\Omega_{0}\left(1+\frac{1}{2} \eta \tan \vartheta \mathrm{J}_{1}(\alpha) \cos \beta\right)
$$

is cyclotron frequency of quasi-particles with the energy spectrum (1) in the magnetic field $\mathbf{H}_{\mathbf{0}}=\left(\mathrm{H}_{0} \sin \vartheta, \mathbf{0}, \mathrm{H}_{0} \cos \vartheta\right), \Omega_{0}=\left(|\mathrm{e}| H_{0} / m c\right) \cos \vartheta, \alpha=\left(m v_{\mathrm{F}} / p_{0}\right) \tan \vartheta, \mathrm{J}_{n}(\alpha)$ is the Bessel function, the initial phase is chosen so that $v_{y}(0)=0$,

$$
v_{\perp}=v_{\mathrm{F}}\left(1-\frac{v_{x}^{(1)}(0)}{v_{\mathrm{F}}}+\frac{\eta p_{0}}{m v_{\mathrm{F}}} \cos (\beta-\alpha)\right)
$$

is the amplitude of the first harmonic of $v_{x}(t)$.

After straightforward calculations the equation (12) can be transformed into the form

$$
\boldsymbol{\Phi}=\int_{-\infty}^{\varphi} \mathrm{d} \varphi^{\prime} \exp \left(\frac{\mathrm{i}}{\Omega} \int_{\varphi^{\prime}}^{\varphi} \mathrm{d} \varphi^{\prime \prime}\left(\tilde{\omega}-\mathbf{k v}\left(\varphi^{\prime \prime}, p_{H}\right)\right)\right)\left(\frac{\mathbf{v}}{\Omega}-\mathrm{i} \frac{\omega}{\Omega} \sum_{p=-\infty}^{\infty} \lambda_{\mathrm{p}} \overline{\boldsymbol{\Phi}}^{(p)} \mathrm{e}^{i p \varphi^{\prime}}\right)
$$

where $\tilde{\omega}=\omega+\mathrm{i} \tau^{-1}$, in the collisionless limit $(\tau \rightarrow \infty)$ we may set $\tilde{\omega}=\omega+\mathrm{i} 0$.

After multiplying the equation (15) by $\mathrm{e}^{-i n \varphi}$ and then integrating with respect to $\beta$ and $\varphi$, we obtain the infinitesimal set of linear equations for the Fourier coefficients 
$\overline{\boldsymbol{\Phi}}^{(p)}$ of the function $\langle\boldsymbol{\Phi}\rangle_{\beta} \equiv 1 /(2 \pi) \int_{-\pi}^{\pi} \mathrm{d} \beta \boldsymbol{\Phi}\left(\varepsilon_{\mathrm{F}}, \beta, \varphi\right)$

$$
\begin{aligned}
\sum_{p} & =\sum_{p=-\infty}^{\infty}\left(\delta_{n p}-\lambda_{\mathrm{p}} \frac{\omega}{\Omega}\left\langle f_{n, p}(\beta)\right\rangle_{\beta}\right) \overline{\mathbf{\Phi}}^{(p)} \\
& =\frac{1}{\Omega}\left\langle\frac{1}{2 \pi} \frac{\int_{0}^{2 \pi} \int_{0}^{2 \pi} \mathrm{d} \varphi \mathrm{d} \varphi_{1} \mathbf{v}\left(\beta, \varphi-\varphi_{1}\right) \mathrm{e}^{-i n \varphi+i R\left(\varphi, \varphi_{1}\right)}}{1-\mathrm{e}^{2 \pi i R(2 \pi, 2 \pi)}}\right\rangle_{\beta}, \\
f_{n, p}(\beta) & =\frac{1}{2 \pi i} \frac{\int_{0}^{2 \pi} \int_{0}^{2 \pi} \mathrm{d} \varphi \mathrm{d} \varphi_{1} \mathrm{e}^{i(p-n) \varphi-i p \varphi_{1}+i R\left(\varphi, \varphi_{1}\right)}}{1-\mathrm{e}^{2 \pi i R(2 \pi, 2 \pi)}} .
\end{aligned}
$$

Here $R\left(\varphi, \varphi_{1}\right) \equiv \Omega^{-1} \int_{\varphi-\varphi_{1}}^{\varphi} \mathrm{d} \varphi^{\prime}\left(\tilde{\omega}-\mathbf{k v}\left(\beta, \varphi^{\prime}\right)\right), \delta_{n p}$, is the Kroneker symbol. The dependence of the cyclotron frequency on $p_{H}$ should be taken into account in the expression $k_{x} v_{x} / \Omega$ in the exponent when $\eta k v_{\mathrm{F}} \gtrsim \Omega$.The coefficients of the Fourier series for the smooth function $L^{\sim}\left(\mathbf{p}, \mathbf{p}^{\prime}\right)$ significantly decrease with their number increasing, so we shall restrict ourselves to taking into account a finite number of the terms in the right part of (16).

The parameter $\eta^{2}$ is usually of the order of $10^{-3}-10^{-5}$. If the inequality $\left(k r_{0}\right)^{-3} \times$ $\left(\eta \omega_{\mathrm{p}} v_{\mathrm{F}} / \Omega \mathrm{c}\right)^{2} \ll 1,\left(r_{0}=v_{\mathrm{F}} / \Omega\right)$ is satisfied, the components of electrical conductivity tensor $\sigma_{z \alpha}, \alpha=x, y$, proportional to $\eta$, and the component $\sigma_{z z} \sim \eta^{2}$ in the dispersion equation can be neglected. Substituting the Fourier series expansion of $\Phi_{i}$ into (7) we obtain

$$
\begin{aligned}
& \sigma_{x x}=\frac{1}{2} \mathrm{e}^{2} v_{\mathrm{F}} \nu\left(\varepsilon_{\mathrm{F}}\right)\left(\bar{\Phi}_{x}^{(1)}+\bar{\Phi}_{x}^{(-1)}\right), \quad \sigma_{y y}=-\frac{1}{2 i} \mathrm{e}^{2} v_{\mathrm{F}} \nu\left(\varepsilon_{\mathrm{F}}\right)\left(\bar{\Phi}_{y}^{(1)}-\bar{\Phi}_{y}^{(-1)}\right), \\
& \sigma_{x y}=\frac{1}{2} \mathrm{e}^{2} v_{\mathrm{F}} \nu\left(\varepsilon_{\mathrm{F}}\right)\left(\bar{\Phi}_{y}^{(1)}+\bar{\Phi}_{y}^{(-1)}\right)=\frac{1}{2 i} \mathrm{e}^{2} v_{\mathrm{F}} \nu\left(\varepsilon_{\mathrm{F}}\right)\left(\bar{\Phi}_{x}^{(1)}-\bar{\Phi}_{x}^{(-1)}\right)
\end{aligned}
$$

Here $\nu\left(\varepsilon_{\mathrm{F}}\right)=m p_{0} / \pi \hbar^{3}$ is the density of states of electrons at the Fermi level. Equations (8), (16), (18) describe the eigenmodes of the electromagnetic field in the Fermi liquid.

Under the strong spatial dispersion $k r_{0} \gg 1, \eta k r_{0} \sim 1$ the integrals about $\varphi, \varphi_{1}$ in the formula (16) can be calculated by means of the stationary phase method, and if $\omega \ll k r_{0}$, the stationary points are determined from the equations $v_{x}(\varphi)=$ $0, v_{x}\left(\varphi-\varphi_{1}\right)=0$. It is easy to see that the maximum component of the tensor $\sigma_{i j}$ is $\sigma_{y y}$ which is proportional to $\left(k r_{0}\right)^{-1}$. The expansion in power series of the components $\sigma_{x \alpha}, \alpha=x, y, z$ begins with the terms of higher order in $\left(k r_{0}\right)^{-1}$. In the basic approximation in small parameters $\left(k r_{0}\right)^{-1}$ and $\eta$ we obtain from (8) the following dispersion equation

$$
\frac{k^{2} c^{2}}{\omega^{2}}=\frac{4 \pi i}{\omega} \sigma_{y y}
$$


The asymptotic expression for $f_{n, p}(\beta)$ for $n, p \ll k r_{0}$ takes the form

$$
\begin{aligned}
f_{n, p}(\beta)= & \frac{1}{k_{x} r_{0}}\left(\cot \frac{\pi}{\Omega}\left(\tilde{\omega}-\langle\mathbf{k v}\rangle_{\varphi}\right) \cos \frac{\pi}{2}(n-p)\right. \\
& \left.+\frac{\sin \left(R\left(\frac{\pi}{2}, \pi\right)-\frac{\pi}{\Omega}\langle\mathbf{k v}\rangle_{\varphi}+\frac{\pi}{2}(n+p)\right)}{\sin \frac{\pi}{\Omega}\left(\tilde{\omega}-\langle\mathbf{k v}\rangle_{\varphi}\right)}\right) .
\end{aligned}
$$

Outside the domain of values of $\omega, \mathbf{k}$ satisfying the condition

$$
\left|\omega-n \Omega_{0}\right|>\max \left|\langle\mathbf{k v}\rangle_{\varphi}\right|
$$

the integrands in the formula (16) have a pole and after integration over $p_{H}$ the dispersion equation gets an imaginary part responsible for the strong absorption of the wave.

After averaging the component of electron velocity over the period of motion along the cyclotron orbit, we obtain

$$
\langle\mathbf{k v}\rangle_{\varphi}=\mathbf{k v}_{\mathbf{B}}=\eta v_{\mathrm{F}} \mathrm{J}_{0}(\alpha)\left(k_{x} \tan \vartheta+k_{z}\right) \sin \beta .
$$

For the directions of $\mathbf{H}_{\mathbf{0}}$ when $\alpha$ is equal to one of the roots $\alpha_{i}=\left(m v_{\mathrm{F}} / p_{0}\right) \tan \vartheta_{i}$ of the equation $\mathrm{J}_{0}(\alpha)=0$, the average $\langle\mathbf{k v}\rangle_{\varphi} \sim \eta^{2}$ is of the second order in $\eta$ and there is no collisionless absorption. Having determined $\overline{\boldsymbol{\Phi}}^{( \pm 1)}$ using (16), we can find the conductivity tensor components. Within the framework of the model which allows for the zeroth and first Fourier harmonics of the Landau function

$$
L\left(\mathbf{p}, \mathbf{p}^{\prime}\right)=L_{0}+2 L_{1} \cos \left(\varphi-\varphi^{\prime}\right),
$$

the dispersion equation becomes

$$
\begin{aligned}
1- & \lambda_{0} \frac{\omega}{\Omega_{0}} \frac{1}{\xi_{0}}\left(\cot \frac{\pi \tilde{\omega}}{\Omega_{0}}+\frac{\left\langle\sin R\left(\vartheta_{i}\right)\right\rangle_{\beta}}{\sin \frac{\pi \tilde{\omega}}{\Omega_{0}}}\right)=2 \frac{\omega}{\Omega_{0}} \frac{1}{\xi_{0}}\left(\left(\frac{\omega_{\mathrm{p}} v_{\mathrm{F}}}{\Omega_{0} c \xi_{0}}\right)^{2}-\lambda_{1}\right) \\
& \times\left(-\cot \frac{\pi \tilde{\omega}}{\Omega_{0}}+\frac{\left\langle\sin R\left(\vartheta_{i}\right)\right\rangle_{\beta}}{\sin \frac{\pi \tilde{\omega}}{\Omega_{0}}}+\frac{\lambda_{0}}{\xi_{0}} \frac{\omega}{\Omega_{0}} \frac{\left(\cos ^{2} \frac{\pi \tilde{\omega}}{\Omega_{0}}-\left(\left\langle\sin R\left(\vartheta_{i}\right)\right\rangle_{\beta}^{2}+\left\langle\cos R\left(\vartheta_{i}\right)\right\rangle_{\beta}^{2}\right)\right)}{\sin ^{2} \frac{\pi \tilde{\omega}}{\Omega_{0}}}\right) .
\end{aligned}
$$

Here

$$
\begin{aligned}
R\left(\vartheta_{i}\right)= & \int_{-\pi / 2}^{\pi / 2} \frac{\mathbf{k v}(\varphi)}{\Omega(\beta)} \mathrm{d} \varphi=2 \frac{k_{x} v_{\perp}}{\Omega(\beta)}-\pi \eta \frac{k_{z} v_{\mathrm{F}}}{\Omega_{0}} \mathrm{H}_{0}\left(\alpha_{i}\right) \cos \beta \\
& +\eta \frac{k_{x} v_{\mathrm{F}}}{2 \Omega_{0}} \tan \vartheta_{i} \cos \beta \sum_{n=1}^{\infty} \frac{\mathrm{J}_{2 n+1}\left(\alpha_{i}\right)}{n(n+1)(2 n+1)}
\end{aligned}
$$


$\mathrm{H}_{0}(\alpha)=(2 / \pi) \int_{0}^{\pi / 2} \mathrm{~d} \varphi \sin (\alpha \cos \varphi)$ is the Struve function, $\xi_{0}=k_{x} r_{0}$.

The equation (24) has the solution in the region of the resonance

$$
\omega=n \Omega_{0}+\triangle \omega, \quad 0<|\triangle \omega|<\Omega_{0}, \quad n=1,2,3 \ldots,
$$

where $n \Omega_{0}$ is the frequency corresponding to the cyclotron resonance of $\mathrm{n}$ order. In quasi-isotropic metals the similar types of waves take place only when $\mathbf{k}$ is perpendicular to the direction of the magnetic field.

In the case when $\left(k r_{0}\right)^{-3}\left(\omega_{\mathrm{p}} v_{\mathrm{F}} / \Omega c\right)^{2} \gg 1$, which is usually realized in the conductors whose charge carriers density is about one electron per atom, the left-hand part of the equation (24) may be neglected. If the inequality $1-\left|\left\langle\sin R\left(\vartheta_{i}\right)\right\rangle_{\beta}\right| \gg \xi_{0}^{-1}$ is satisfied, the spectrum for the cyclotron waves can be found in the analytical form

$$
\begin{aligned}
\triangle \omega & =\Delta \omega_{0}+\Delta \omega_{1} \\
\triangle \omega_{0} & =\Omega_{0}\left(\frac{1}{2}-\frac{(-1)^{n}}{\pi} \arcsin \left\langle\sin R\left(\vartheta_{i}\right)\right\rangle_{\beta}\right), \\
\triangle \omega_{1} & =\frac{\Omega_{0} \lambda_{0}}{\pi \xi_{0}}\left(n+\frac{\triangle \omega_{0}}{\Omega_{0}}\right) \frac{\left.\left\langle\cos R\left(\vartheta_{i}\right)\right\rangle_{\beta}^{2}\right)}{1-\left\langle\sin R\left(\vartheta_{i}\right)\right\rangle_{\beta}^{2}} .
\end{aligned}
$$

In the other limited case $\left(k r_{0}\right)^{-3}\left(\omega_{\mathrm{p}} v_{\mathrm{F}} / \Omega c\right)^{2} \ll 1$, the solution of $(24)$ can be represented in the form (26) with:

$$
\begin{aligned}
\triangle \omega & =\frac{n \Omega_{0}}{\pi \xi_{0}}\left(a \pm \sqrt{a^{2}+2\left(\gamma-\lambda_{1}\right) \lambda_{0}\left(1-\left\langle\sin R\left(\vartheta_{i}\right)\right\rangle_{\beta}^{2}-\left\langle\cos R\left(\vartheta_{i}\right)\right\rangle_{\beta}^{2}\right)}\right), \quad(28) \\
a & =\frac{1}{2}\left[\lambda_{0}-2\left(\gamma-\lambda_{1}\right)+(-1)^{n}\left(\lambda_{0}+2\left(\gamma-\lambda_{1}\right)\right)\left\langle\sin R\left(\vartheta_{i}\right)\right\rangle_{\beta}\right], \quad \gamma=\left(\frac{\omega_{\mathrm{p}} v_{\mathrm{F}}}{\Omega_{0} c \xi_{0}}\right)^{2} .
\end{aligned}
$$

If $\eta k v_{\mathrm{F}} \ll \Omega_{0}$, the spatial dispersion along the z-direction is not essential, $R\left(\vartheta_{i}\right)=$ $2 k_{x} r_{0}$ and formulas $(27),(28)$ transform into the expressions obtained in [14] for the case when the wave vector $\mathbf{k}=(k, 0,0)$ is orthogonal to an external magnetic field. 


\section{References}

1. Wosnitza J., Springer Tracts in Mod. Phys., 1996, 136, 1.

2. Kartsovnik M.V., Laukhin V.N., J. Phys. I., 1996, 6, 1753.

3. Singleton J., Rep. Prog. in Phys., 2000, 63, 1111.

4. DemishevS.V., SemenoA.V., Sluchanko N.E., Samarin N.A., Pis'ma Zh. Eksp. Teor. Fiz., 1995, 61, 299 (in Russian); JETP Lett. 1995, 61, 313.

5. Demishev S.V., Semeno A.V., Sluchanko N.E., et al., Phys. Rev. B, 1996, 53, 12794.

6. Demishev S.V., Semeno A.V., Sluchanko N.E., et al., Zh. Eksp. Teor. Fiz., 1997, 111, 979 (in Russian); JETP, 1997, 84, 540.

7. Platzman P.M., Wolf P.A. Waves and Interactions in Solid State Plasma. Academic, New York, 1973.

8. Kirichenko O.V., Peschansky V.G., Stepanenko D.I., Zh. Eksp. Teor. Fiz., 2004, 126, 1435 (in Russian); JETP, 2004, 99, 1253.

9. Landau L.D., Zh. Eksp. Teor. Fiz., 1956, 30, 1058 (in Russian); JETP, 1956, 3, 920.

10. Silin V.P., Zh. Eksp. Teor. Fiz., 1957, 33, 495 (in Russian); JETP, 1958, 6, 387.

11. Silin V.P., Zh. Eksp. Teor. Fiz., 1958, 35, 1243 (in Russian); JETP, 1959, 8, 870.

12. Schultz S., Dunifer G., Phys. Rev. Lett., 1967, 18, 283.

13. Abrikosov A.A. Fundamentals of the Theory of Metals. North-Holland, Amsterdam, 1988.

14. Kirichenko O.V., Peschansky V.G., Stepanenko D.I., Phys. Rev. B, 2005, 71, 045304. 


\section{Високочастотні циклотронні моди у Фермі рідині Q2D шаруватих провідників}

\section{О.В.Кириченко, В.Г.Піщанський, Д.І.Степаненко}

Фізико-Технічний Інститут Низьких температур ім. Б.І.Веркіна НАН України, Україна, 61164, м. Харків, пр. Леніна, 47

Отримано 18 липня 2005 р.

Теоретично досліджено поширення електромагнітних хвиль у шаруватих провідниках у присутності зовнішнього магнітного поля. При деяких орієнтаціях магнітного поля щодо шарів провідника беззіткнене поглинання відсутнє і поширення слабозгасаючих колективних мод є можливим навіть в умовах сильної просторової дисперсії. У короткохвильовій межі можливе існування електромагнітних хвиль з частотами біля резонансів при довільній орієнтації хвильового вектора щодо зовнішнього магнітного поля. Знайдено спектр хвиль з частотами біля кратних циклотронних резонансів з врахуванням Фермі-рідинної взаємодії електронів провідності.

Ключові слова: шаруваті провідники, Фермі-рідина, циклотронні хвилі

PACS: $72.15 . N j, 72.30 .+q$ 